\title{
A Practical Method for Optimized Reactive Power Sharing Among Passive Filters
}

\author{
M. R. Vatani, D. Solati Alkaran, M. J. Sanjari and G. B. Gharehpetian \\ Electrical Engineering Department \\ Amirkabir University of Technology \\ 15914 Tehran (Iran) \\ Phone/Fax number: +98 21 66406469, e-mail: mvatani@aut.ac.ir, davoodsolati@aut.ac.ir, mjsanjari@aut.ac.ir, \\ grptian@aut.ac.ir
}

\begin{abstract}
This paper presents a practical method for reactive power sharing among harmonics filters. The proposed method minimizes the total harmonic distortion of the current. The current is measured at the incoming bus to which harmonics filters are connected. The total harmonics distortion and individual harmonics of the voltage at the bus to which the harmonic filters are connected and the individual harmonics of the current measured at the mentioned measurement point are used as constraints of optimization. The Optimization Toolbox of MATLAB software is used in this paper. The simulation results show the efficiency and robustness of the proposed method.
\end{abstract}

\section{Key words}

Harmonics filter, Reactive power sharing, Resonance frequency, Total harmonic distortion.

\section{Introduction}

Due to non-linear behaviour of loads in power networks, the utility grid voltage is diverted from its pure sinusoidal shape at the fundamental frequency, although this signal is repetitive at the fundamental frequency. Non-linear loads generate harmonic currents and voltages. Some advanced techniques such as magnetic flux compensation, series/shunt active filter systems, and harmonic current injection are introduced to decrease harmonic problems in power systems. However, because of their complexity and high cost, they have not been practical solutions and cannot compete with passive harmonic filter systems. The passive harmonic filters are installed in the industry field and applied to a wide range of applications.

The passive harmonic filters have a low cost, simple design and reliable operation. Therefore, they have been widely used for harmonics mitigation in power systems [13]. The single-tuned filters are the most commonly used passive filters. In comparison with other methods, these filters are simple and the least expensive one [4-6]. The LC series filter is the most common and inexpensive type of passive filters. This filter is connected in shunt with the main distribution networks and should have low impedance to a tuned particular harmonic frequency. Therefore, the harmonic currents flow through the filter which has the least impedance path. Selecting the

appropriate value for capacitor is one of the most essential steps for designing single tuned filters to result in appropriate power factor correction at fundamental frequency. Figure 1 shows the circuit diagram of different types of passive filters.

The first step of designing a harmonics filter is the determination of the reactive power that it should generate at network nominal frequency. The impedancefrequency of harmonics filters depends on their reactive power capacity. Therefore, the reactive power sharing among passive harmonics filters is one of the most important issues in designing procedures.

According to the IEEE filter design procedure [7] depicted in Figure 2, the capacitor voltage and capacity and the reactor impedance are needed as predetermined values. Therefore, it can be time consuming to reach to acceptable values if the initial values of the mentioned parameters have not been estimated appropriately. Thus, this algorithm needs expert persons to choose initial values for design parameters. The PC-based computer programs can be considered as a useful tool for designing harmonic filters and harmonic analysis [8].

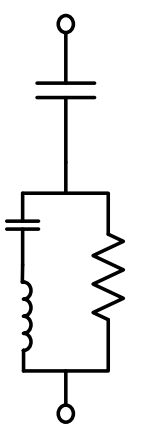

C-Type High Pass

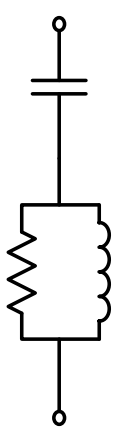

High Pass

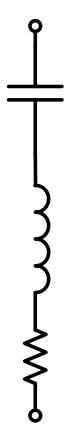

Single Tuned

Fig. 1. Different types of first order of passive filters

This paper presents a practical method for the determination of the reactive power sharing among the single tuned filters. This method is based on the optimization using the impedance of single tuned harmonic filters and network parameters. Hence, it 
guarantees the appropriate power factor correction and harmonic current elimination. Moreover, considering the capacitor voltage and capacity and the reactor impedance as design parameters, there is no need for a trial-and-error approach. This paper is organized as follows: the proposed method is discussed in the next section. The simulation results and discussion are presented and drawn in section 3 and conclusions are presented in the last section.

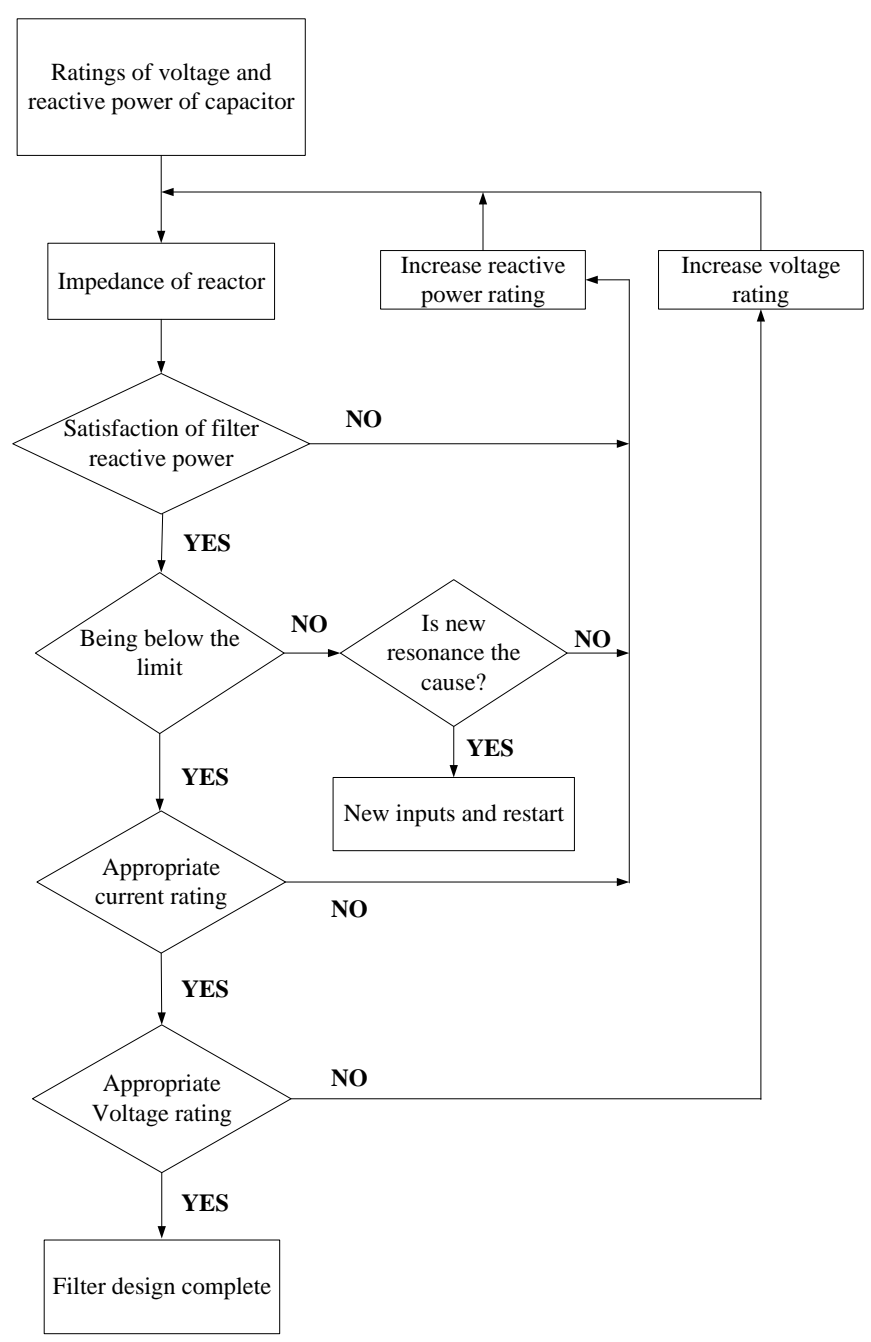

Fig. 2. IEEE harmonic filter design procedure

\section{Proposed Method}

Different methods for reactive power sharing among harmonics filters have different impedance-frequency characteristics for the bus to which the filters are connected. The impedance-frequency characteristic is the most important concept in harmonics filter design.

As shown in Figure 3, the generated the harmonics by harmonic source have two general paths: toward upstream network and harmonic filters. The best operation of harmonic filters can be achieved when the minimum value of generated harmonics flows toward upstream network. In other words, harmonic filters should absorb the maximum values of generated harmonics.

The proposed method minimizes the current total harmonic distortion. The measurement point of the current is shown in Figure 3. Firstly, the equations of the impedance of the single tuned harmonic filters should be calculated in terms of input parameters [9]. For instance, this equation for a single tuned harmonic filter is, as follows:

$$
\begin{aligned}
& Z=R+j 2 \pi f L+\frac{1}{j 2 \pi f C} \\
& R=\frac{n V^{2}}{Q Q_{f}\left(n^{2}-1\right)} \\
& L=\frac{R Q_{f}}{n 2 \pi f_{s}} \\
& C=\frac{1}{n R Q_{f} 2 \pi f_{s}}
\end{aligned}
$$

where:

$R$ : Resistance of single tuned filter

$L$ : Reactance of single tuned filter

$C$ : Capacitance of single tuned filter

$f$ : Fundamental frequency

$n$ : Tuning frequency

$V$ : Bus voltage

$Q$ : Reactive power of single tuned filter

$Q_{f}$ : Quality factor of single tuned filter

$f_{s}$ : Series resonance frequency

In order to prevent from using a trial-and-error approach, tuning frequency and quality factor are considered as design parameters. Furthermore, using PC-based computer program for the optimization of the total harmonic distortion at the mentioned point results in accurate and robust results.

The current and the voltage total harmonic distortion are considered as follows:

$\begin{aligned} T H D_{I} & =\frac{\sum_{k=2}^{n} I_{k}^{2}}{I_{1}} \\ T H D_{V} & =\frac{\sum_{k=2}^{n} V_{k}^{2}}{V_{1}}\end{aligned}$

where, $T H D_{I}$ and $T H D_{V}$ are current and the voltage total harmonic distortion, respectively and $I_{K}$ is the $k^{\text {th }}$ order current harmonic rms value as well as $V_{K}$ is the $k^{\text {th }}$ order voltage harmonic rms value. Also, $I_{l}$ and $V_{l}$ are the current and voltage values $\mathrm{rms}$ at the fundamental frequency, respectively. Then, the current total harmonic distortion is minimized by using the Optimization Toolbox of MATLAB software subject to:

- $\Sigma Q_{i}=Q_{\text {Total }}$

- $T H D_{V}<$ allowable value

- $H D_{V}<$ allowable value

- $H D_{I}<$ allowable value 
where, $Q_{\text {Total }}$ and $Q_{i}$ are the total reactive power of harmonics filters and reactive power of each harmonic filters, respectively. Also, $H D_{V}$ and $H D_{I}$ are the individual harmonic distortion for the measured voltage and current signals. The allowable values also can be determined according to standards or recommendations of expert engineers. Due to using harmonic load flow based on the impedance value of each harmonic filter (expressed in terms of its reactive power generation and given network parameters) the output of the optimization program is the contribution of each harmonic filter in producing the total reactive power.

The proposed method not only minimizes the injected total harmonic distortion to upstream network, but also considers the acceptable range of the individual harmonics distortion of the measured current and voltage. It also reduces the probability of the parallel resonance by checking the range of the total and individual harmonic distortion which should be in allowable ranges.

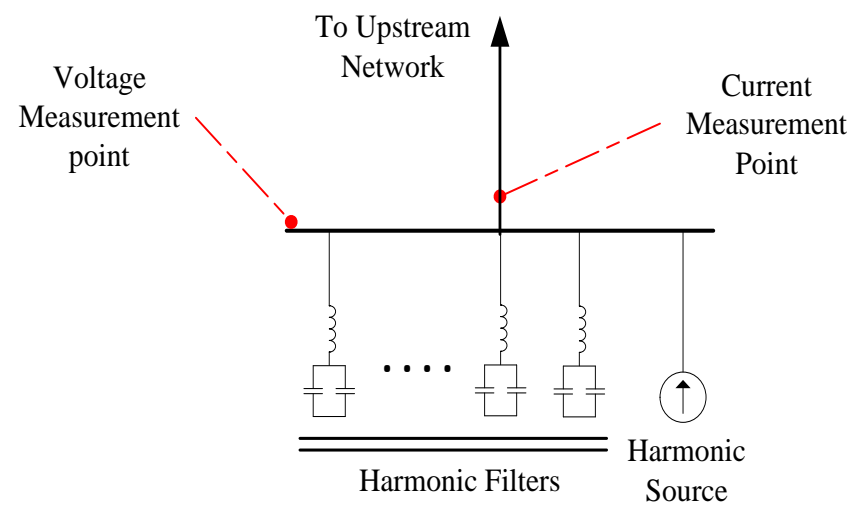

Fig. 3. Current and voltage measurement point

\section{Simulation Results}

Figure 4 shows the test network. The network has four filters for $1^{\text {st }}, 2^{\text {nd }}, 3^{\text {rd }}$ and $4^{\text {th }}$ harmonics. The total reactive power of harmonic filters is $120 \mathrm{MVAR}$ and they are connected to the $33 \mathrm{kV}$ bus. The voltage and current are measured at this bus and the secondary side of the transformer, respectively. The harmonic orders generated by harmonic source are listed in Table I.

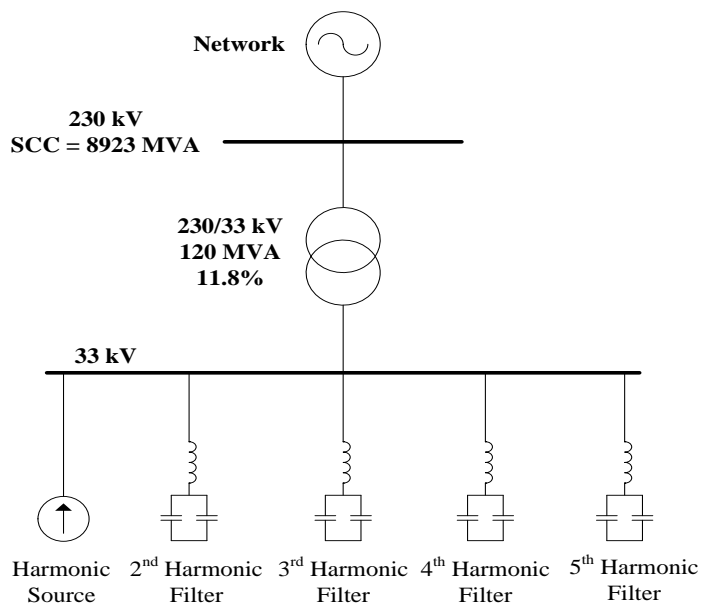

Fig. 4. Test network
Table I. Generated Harmonic Values

\begin{tabular}{|c|c|c|c|}
\hline $\begin{array}{c}\text { HARMONIC } \\
\text { ORDER }\end{array}$ & $\begin{array}{c}\text { CURRENT } \\
(\mathrm{A})\end{array}$ & $\begin{array}{c}\text { HARMONIC } \\
\text { ORDER }\end{array}$ & $\begin{array}{c}\text { CURRENT } \\
(\mathrm{A})\end{array}$ \\
\hline 1 & 2000 & 9 & 66 \\
\hline 2 & 148 & 10 & 12 \\
\hline 3 & 370 & 11 & 36 \\
\hline 4 & 72 & 12 & 7 \\
\hline 5 & 200 & 13 & 23 \\
\hline 6 & 35 & 15 & 23 \\
\hline 7 & 100 & 17 & 15 \\
\hline 8 & 22 & 19 & 13 \\
\hline
\end{tabular}

The fundamental frequency of the test network is equal to $50 \mathrm{~Hz}$. The network is simulated by using the MATLAB software. The single tuned filters and harmonic source have been modelled as fix impedance and current source, respectively in order to minimize the injected total harmonic distortion to upstream network. This program has been run based on the proposed method expressed in previous section. Considering the contribution of the reactive power of each harmonic filter, the design parameters are calculated by this program. Table II gives the simulation results for the shared reactive power among harmonic filters and their specifications. Table III gives the simulation results of different current and voltage harmonics at the measuring points based on harmonic load flow. Table IV lists the IEEE Standard 1519 [10] values for limits of voltage and current harmonics. Comparing the standard values and the simulation results, it can be said that the voltage and current total harmonic distortions as well as individual harmonic distortions are less than the allowable values. By using the proposed optimization, not only the injected total harmonic distortion to upstream network not only is in permissible range, but also it has been minimized.

Table II. Specification of Harmonic Filters

\begin{tabular}{|l|c|c|c|c|}
\hline FILTER ORDER & 2 & 3 & 4 & 5 \\
\hline REACTIVE POWER $($ MVAR) & 45 & 45 & 15 & 15 \\
\hline TUNING FREQUENCY $(\mathrm{Hz})$ & 97 & 145.5 & 194 & 247.5 \\
\hline CAPACITANCE $(\mu \mathrm{F})$ & 94.48 & 116 & 40.93 & 41.98 \\
\hline INDUCTANCE $(\mathrm{mH})$ & 27.87 & 10.31 & 16.44 & 10.26 \\
\hline QUALITY FACTOR & 30 & 100 & 100 & 200 \\
\hline
\end{tabular}

Table III. Current and Voltage Harmonics

\begin{tabular}{|c|c|c|}
\hline $\begin{array}{c}\text { HARMONIC } \\
\text { ORDER }\end{array}$ & $\begin{array}{c}\text { CURRENT } \\
\text { HARMONICS }(\%)\end{array}$ & $\begin{array}{c}\text { VOLTAGE } \\
\text { HARMONICS }(\%)\end{array}$ \\
\hline THD & 4.98 & 3.62 \\
\hline 2 & 2.83 & 0.72 \\
\hline 3 & 2.69 & 1.03 \\
\hline 4 & 0.71 & 0.36 \\
\hline 5 & 1.15 & 0.74 \\
\hline 6 & 0.56 & 0.43 \\
\hline 7 & 1.9 & 1.7 \\
\hline 8 & 0.47 & 0.48 \\
\hline 9 & 1.4 & 1.61 \\
\hline 10 & 0.26 & 0.33 \\
\hline 11 & 0.82 & 1.15 \\
\hline 12 & 0.16 & 0.25 \\
\hline 13 & 0.51 & 0.85 \\
\hline 15 & 0.49 & 0.36 \\
\hline 17 & 0.35 & 0.74 \\
\hline & & \\
\hline
\end{tabular}


Table IV. IEEE Standard Limits for Current and Voltage Harmonics

\begin{tabular}{|l|c|c|c|}
\cline { 2 - 4 } \multicolumn{1}{c|}{} & \multicolumn{2}{c|}{$\begin{array}{c}\text { INDIVIDUAL HARMONIC } \\
\text { DISTORTION }(\%)\end{array}$} & $\begin{array}{c}\text { THD } \\
(\%)\end{array}$ \\
\hline \multirow{2}{*}{ CURRENT } & $\mathrm{h}<11$ & $11 \leq \mathrm{h}<17$ & 5 \\
\cline { 2 - 4 } & 4 & 4 & 5 \\
\hline VOLTAGE & \multicolumn{2}{|c|}{3} & 5 \\
\hline
\end{tabular}

The impedance-frequency characteristic of harmonic filters and the harmonic amplification at the $33 \mathrm{kV}$ bus are shown in Figure 5 and Figure 6, respectively. Figure 5 indicates that each filter has minimum impedance value at its resonance frequency. According to Figure 6, the parallel resonance frequencies and harmonic amplification frequencies are far from operating frequencies of harmonic filters. Therefore, the probability of the parallel resonance has been minimized by optimizing the injected total harmonic distortion to upstream network.

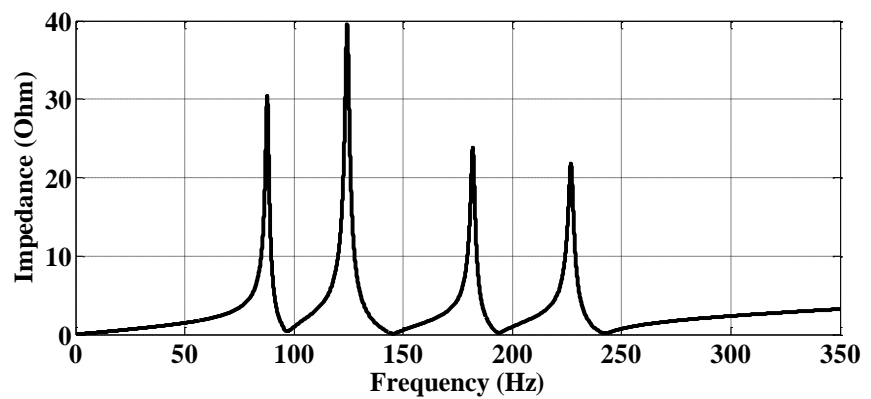

Fig. 5. Impedance-frequency characteristic of harmonic filters

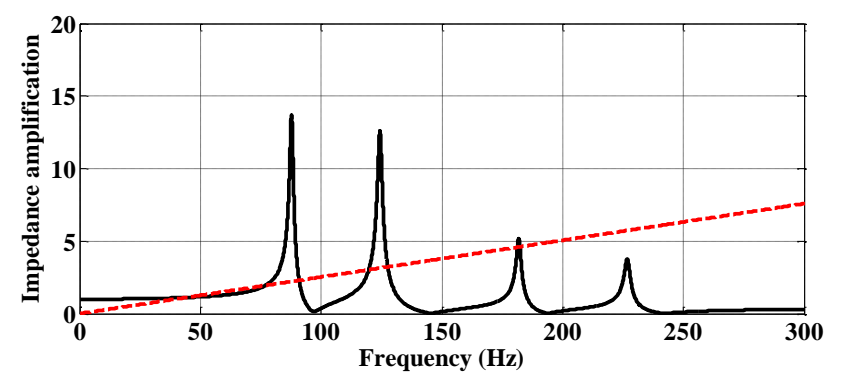

Fig. 6. Harmonic amplification

\section{Conclusion}

A practical method for reactive power sharing among passive filters has been proposed in this paper. This method minimizes the total harmonic distortion of the current measured at the incoming of the bus to which filters have been connected. The optimization has constraints for reliable operation of filters. This method not only corrects the power factor appropriately, but also decreases the probability of the parallel resonance by checking the range of the total and individual harmonic distortion which should be in allowable ranges. The simulation results confirm the effectiveness of the proposed method.

\section{References}

[1] Z. A. Memon, M. A. Uqaili and M. A. Soomro, "Experimental Analysis of Harmonic Mitigation Efforts of Three-Phase Six-Pulse Controller by Using Pass Filters",
Mehran University Research Journal of Engineering \& Technology, Volume 30, pp. 653-656.

[2] J. C. Das, "Passive Filters-Potentialities and Limitations", IEEE Transactions on Industry Applications, Volume 40, pp. 232-241, 2004.

[3] R. Klempka, "Designing a group of single-branch filters", Electrical Power Quality and Utilization, EPQU'03, September 17-19 2003, Krakow, Poland.

[4] S. C. Young, and C. Hanju, "A Single-Tuned Passive Harmonic Filter Design Using Transfer Function Approach for Industrial Process Application", International Journal of Mechatronics and Automation, Volume 1, pp. 90-96.

[5] R. Noroozian and G. B. Gharehpetian, "An Investigation on Combined Operation of Active Power Filter with Photovoltaic Arrays", International Journal of Electrical Power and Energy Systems, Vol. 46, pp. 392-399.

[6] A. B. Nassif, W. Xu, W. Freitas "An Investigation on the Selection of Filter Topologies for Passive Filter Applications", IEEE Transactions on Power Delivery, vol. 24, July 2009.

[7] D. A. Gonzalez and J. C. McCall, "Design of filters to reduce harmonic distortion in industrial power systems," IEEE Trans. Ind. Application, vol. IA-23, pp. 504-511.

[8] J. R. Ramos, A. Marani, A. Cavallini, and Loggini, "Simplified harmonic simulation procedure," in Conf. Rec. IEEE-IAS Annu. Meeting, 1993, vol. 2, pp. 1594-1600.

[9] Dugan R., McGranaghan M., "Electrical power systems quality", McGraw-Hill, 2002.

[10] IEEE Std 519-1992, "IEEE recommended practices and requirements for harmonic control in electrical power systems". 